\title{
Coronavirus variant COVID-19 pandemic: a report to seafarers
}

\author{
Paolo Sossai ${ }^{1,2}$, Silvia Uguccioni ${ }^{3}{ }^{\oplus}$, Giuseppe Sandro Mela $^{4}{ }^{(1)}$, \\ Marzio DiCanio ${ }^{2}$, Francesco Amenta ${ }^{1,2}$ \\ ${ }^{1}$ Department of Drug Sciences and Health Products, University of Camerino, Italy \\ ${ }^{2}$ Department of Research, Centro Internazionale Radio Medico (C.I.R.M.), Rome, Italy \\ ${ }^{3}$ Ministry of Public Education, Pesaro, Italy \\ ${ }^{4}$ Department of Medicine, University of Genoa, Italy
}

\begin{abstract}
We report the current situation regarding the COVID-19 pandemic with particular regard to seafarers and with the indications drawn up by the Centro Internazionale Radio Medico (C.I.R.M.) in this regard.

Key words: coronavirus, COVID-19, maritime medicine, acute distress respiratory syndrome, occupational health
\end{abstract}

(Int Marit Health 2020; 71, 3: 191-194)

\section{INTRODUCTION}

Coronaviruses are a large family of viruses, which include 4 genera: $\alpha$ and $\beta$ infect mammals while $\gamma$ and $\delta$ infect birds. In humans, several coronaviruses are known to cause respiratory infections ranging from the common cold to more severe diseases, such as severe acute respiratory syndrome (SARS) and Middle East respiratory syndrome (MERS) [1]. The most recently discovered coronavirus is SARS-CoV-2 which causes the coronavirus disease named COVID-19. This new virus and resulting disease were unknown before the outbreak that began in Wuhan, China, in November-December 2019. SARS-CoV-2 and SARS-CoV share approximately $80 \%$ of genomic material.

This report takes stock of the situation approximately 3 months after it was declared a pandemic by the World Health Organization (WHO; March 11, 2020) [2], and reports the position of the Centro Internazionale Radio Medico (C.I.R.M., International Radio Medical Centre, the Italian Telemedical Advice Service) with regard to this topic. The C.I.R.M. was established in 1935 to provide medical care via telecommunication systems to seafarers of all nationalities, sailing across the seas, aboard vessels without a doctor on board.

The ongoing COVID-19 outbreak probably started in November-December 2019. Wuhan is the capital of the Hubei Province, located at the confluence of the Changjiang
River and the Hanshui River. Today Wuhan is considered the political, economic, financial, commercial, cultural and educational centre of central China. It is a major transportation hub, with dozens of railways, roads and expressways passing through the city and connecting to other major cities. Wuhan Tianhe International Airport, which opened in April 1995 in order to replace the previous Hankou Wangjiadun and Nanhu airports, thus becoming Wuhan's major airport, is one of the busiest airports in central China. Wuhan is also a major hub for maritime transport in central China. The port of Wuhan hosts services for the local population and for shipping enterprises.

The John Hopkins University database, in the situation report of June 28, 2020, stated that there are $9,953,083$ confirmed cases with 498,217 deaths [2]. There are 240,136 confirmed cases in Italy with 34,716 deaths, with a lethality rate of approximately $14 \%$ [3].

The signs and symptoms of 2019-nCoV include fever, cough, respiratory problems and pneumonia. Human-to-human transmission may occur during the incubation period (before symptoms appear), and the average time from infection until the onset of the disease is approximately 7 days, with a maximum incubation period of approximately 14 days. WHO has defined suspect, probable and confirmed cases, as well as contacts, as indicated below [4]. 


\section{SUSPECT CASE}

- A patient with acute respiratory illness (fever and at least one sign/symptom of respiratory disease, e.g., cough, shortness of breath), AND a history of travel to or residence in a location reporting community transmission of COVID-19 disease during the 14 days prior to symptom onset; OR

- A patient with any acute respiratory illness AND having been in contact with a confirmed or probable COVID-19 case (see definition of contact) in the last 14 days prior to symptom onset; OR

- A patient with severe acute respiratory illness (fever and at least one sign/symptom of respiratory disease, e.g., cough, shortness of breath; AND requiring hospitalisation) AND in the absence of an alternative diagnosis that fully explains the clinical presentation.

\section{PROBABLE CASE}

- A suspect case for whom testing for the COVID-19 virus is inconclusive; OR

- A suspect case for whom testing could not be performed for any reason.

\section{CONFIRMED CASE}

A person with laboratory confirmation of COVID-19 infection, irrespective of clinical signs and symptoms. See laboratory guidance for details: https://www.who.int/emergencies/diseases/novel-coronavirus-2019/technicalguidance/laboratory-guidance.

\section{CONTACT}

A contact is a person who experienced any one of the following exposures during the 2 days before and the 14 days after the onset of symptoms of a probable or confirmed case:

- face-to-face contact with a probable or confirmed case within $1 \mathrm{~m}$ and for more than $15 \mathrm{~min}$;
- direct physical contact with a probable or confirmed case;

- direct care for a patient with probable or confirmed COVID-19 disease without using proper personal protective equipment;

- other situations as indicated by local risk assessments. Note: For confirmed asymptomatic cases, the period of contact is measured as the 2 days before through the 14 days after the date on which the sample was taken which led to confirmation.

A recent review of 22 studies by Krampf et al. [5], on SARS, MERS and HcoV (alphacoronavirus) syndromes, all variants of coronavirus, has highlighted the persistence of these viruses on inanimate surfaces for up to 9 days, and that the use of ethanol, sodium hypochlorite and hydrogen peroxide inactivates the viruses within one minute. In the recent history of public hygiene we have seen the dangerousness of the coronavirus family variants, namely the SARS and MERS viruses. The main mode of transmission of these pathologies was also airborne, with COVID-19 having similar animal reservoirs as SARS [1]; while the main animal reservoir for MERS seems to have been the camel [1]. In 2003 the fatality rate of SARS, which also originated in China, was approximately $10 \%$, while in 2012 the fatality rate of MERS was an average of approximately $35 \%$ (WHO data). The initial clinical information on COVID-19 is summarised in Table 1 , which reports the data of two initial patient cohorts from two hospitals in Wuhan $[6,7]$ and subsequently one cohort.

The initial data showed that almost all patients had a fever (98\% of cases) and radiographically visible pneumonia (100\% of cases). A cough was present in $60-76 \%$ of cases and dyspnoea in 30-55\% of cases. The median time between the onset of symptoms and hospitalisation was 7 days for both of the patient cohorts reported in Table 1 . If acute respiratory distress syndrome developed, the median time to onset was 8-9 days from the start of symptoms. This data has been substantially confirmed

Table 1. Patient data from two hospitals in Wuhan (two initial patient cohorts)

\begin{tabular}{lll}
\hline & $\begin{array}{l}\text { Huang C et al. [6] } \\
\text { (Jin Yintan Hospital, Wuhan): } \\
\text { 41 patients (January 2-24, 2020) }\end{array}$ & $\begin{array}{l}\text { Wang D et al. [7] } \\
\text { (Zhongnan Hospital, Wuhan): } \\
\text { 138 patients (January 1-28, 2020) }\end{array}$ \\
\hline Men & $73 \%$ & $54.3 \%$ \\
Mean age & 49 years & 56 years \\
Main symptoms & Fever (98\%), cough (76\%), dyspnoea (55\%), & Fever (98.6\%), dry cough (59.4\%), dyspnoea (31.2\%), \\
& lymphopenia (63\%) & $100 \%$ \\
Pneumonopenia (70.3\%) \\
Comorbidities & $100 \%$ & $46.4 \%$ \\
Intensive Care Unit & $32 \%$ & $26.1 \%$ \\
Lethality & $32 \%$ & $4.3 \%$
\end{tabular}


with a meta-analysis by Li et al. [8] with 1994 patients originating from 10 scientific works selected. The lethality of this meta-analysis was $7 \%$. The largest cause for the transmission and the wide spread of COVID-19 within the province of Hubei was local transportation, while the spread to other countries was through airplanes. Maritime transport could have, or has, played a role in the spreading of COVID-19, but given the slower speed and the confined spaces used, COVID-19 spreads less effectively than with air transport.

The first American case of COVID-19 was reported on March 5, 2020, and in addition to the symptoms summarised in Table 1, the case also presented with abdominal pain and loose stools, testing positive for COVID-19 [9]. On June 28, 2020 the confirmed cases of SARS-CoV-2 in United States are 2,510,092 with 125,539 deaths [3].

The term quarantine, or forced confinement, is wellknown in the maritime sector and actually originates from the word "forty" in Italian, which refers to the forty day period during which the Venetian government in the $16^{\text {th }}$ century forbade any ships from approaching the port of Venice in order to avoid contaminating the city with the black plague. Sikorska [10] highlights that the lack of an effective treatment or a vaccine renders quarantine as the only effective protection.

In the maritime sector there are two types of shipping that must be underline. Cruise ships carry a large number of people, travelling to multiple destinations and providing a high number of available services (including healthcare ones). Cargo ships, instead, transport any type of goods (hydrocarbons, metals, various chemicals, etc.) and the crew does not exceed 30 members, without any medical services and with an average 3-6 months period of stay on board. In order to be able to continue supplying raw materials (hydrocarbons, chemical substances, etc.) even in endemic areas, this type of commercial maritime transport must continue, with the foresight that the personnel on board cannot disembark and no personnel can be replaced. Reducing all contact between the ship and external personnel to a minimum is essential, and with these dealings a safe distance must be kept, and protective gloves and masks must be used as specified by the guidelines issued by the United States Centre for Disease Control and Prevention [11]. According to these guidelines, the safety distance on board should be approximately 6 feet (approx. $1.8 \mathrm{~m}$ ).

The use of N95 filtering face masks is only recommended for those who are directly assisting suspect/probable or confirmed cases, and this assistance should always be provided by the same people, who will also wear disposable goggles as protection and medical gloves. For individuals experiencing fever, cough or dyspnoea, only the use of a surgical mask is recommended.
The frequent washing of hands for at least $20 \mathrm{~s}$, with water and soap or a $60-95 \%$ alcohol solution, is prescribed to everyone.

In general, the medical devices on these ships are limited to only a sphygmomanometer and a thermometer, thus lacking the ability of evaluating fundamental vital signs such as blood oxygen saturation or performing an electrocardiogram test.

In this respect, the C.I.R.M. has always insisted on diffusing telemedicine stations on merchant ships, which would render it possible to quickly assess the conditions of seafarers on board, even remotely.

The issues encountered aboard these types of ships during this COVID-19 epidemic are:

- discerning between a common flu and a form of COVID-19;

- on-board isolation methods;

- any method of evacuation (MEDical EVACuation).

In the event of a suspect/probable case of COVID-19, knowing the history, movements and origin of the index case(s) is absolutely necessary. With this information and with the epidemiological data that is being collected, the case can be categorised in the appropriate risk range. It is essential to follow the clinical progress of the patient(s) who, if suspected to have contracted COVID-19, should be isolated in the infirmary, which should be equipped with one or two hospital beds and medical oxygen, and where the use of the toilet is reserved to the patient(s) and can be easily cleaned with a sodium hypochlorite solution.

If the fever $\left(\geq 38^{\circ} \mathrm{C}\right)$ and respiratory symptoms resolve within a few days and the risk of having been infected is low, clearly, chances are the person did not contract COVID-19. Nevertheless, the lingering issue is that COVID-19 can also manifest itself in milder forms, similar to the flu, and diagnostic tests for COVID-19 cannot be conducted in all the ports. In the event of a suspected COVID-19 infection, the staff must obviously undergo quarantine and therefore cannot leave the ship that has docked at the port, to load and unload goods.

In ports of entry, the ship with suspect cases should be moored and the parties should disembark and undergo diagnostic tests in local equipped healthcare facilities, if possible. If such tests are not available, the index case will still have to undergo radiological diagnostics in order to check if viral pneumonia is present. If the diagnostic tests for the index case are not available, the personnel on board should remain in isolation for 14 days, starting from when the symptoms of the index case began. Frequent hand washing is a requirement for all quarantined personnel. As of today, potential COVID-19 vaccines are still in the testing phase, and with time being of the essence, many companies 
have announced that they have been developing potential vaccines to curb further spread of the virus.

\section{ACKNOWLEDGEMENTS}

This study was supported by the ITF Trust grant No. $1508 / 2020$ to Centro Internazionale Radio Medico (C.I.R.M.). Institutional funding of the University of Camerino, Italy, supported also this work.

\section{REFERENCES}

1. Perlman S, McIntosh K. Coronaviruses, including Severe Acute Respiratory Syndrome (SARS) and Middle East Respiratory Syndrome (MERS). In: Mandell, Douglas, and Bennett's Principles and Practice of Infectious Diseases. Elsevier, Philadelphia 2020.

2. https://www.epicentro.iss.it/en/coronavirus/sars-cov-2-international-outbreak (June 28, 2020).

3. https://coronavirus.jhu.edu/map.html (June 28, 2020).

4. https://www.who.int/emergencies/diseases/novel-coronavirus-2019/technical-guidance/surveillance-and-case-definitions..

5. Kampf G, Todt D, Pfaender S, et al. Persistence of coronaviruses on inanimate surfaces and their inactivation with biocidal agents. J Hosp
Infect. 2020; 104(3): 246-251, doi: 10.1016/j.jhin.2020.01.022, indexed in Pubmed: 32035997.

6. Huang C, Wang Y, Li X, et al. Clinical features of patients infected with 2019 novel coronavirus in Wuhan, China. Lancet. 2020; 395(10223): 497-506, doi: 10.1016/S0140-6736(20)30183-5, indexed in Pubmed: 31986264.

7. Wang D, Hu Bo, Hu C, et al. Clinical characteristics of 138 hospitalized patients with 2019 novel coronavirus-infected pneumonia in Wuhan, China. JAMA. 2020 [Epub ahead of print], doi: 10.1001/ jama.2020.1585, indexed in Pubmed: 32031570.

8. Li LQ, Huang T, Wang YQ, et al. COVID-19 patients' clinical characteristics, discharge rate, and fatality rate of meta-analysis. J Med Virol. 2020; 92(6): 577-583, doi: 10.1002/jmv.25757, indexed in Pubmed: 32162702.

9. Holshue ML, DeBolt C, Lindquist S, et al. Washington State 2019$n$ CoV Case Investigation Team. First Case of 2019 Novel Coronavirus in the United States. N Engl J Med. 2020; 382(10): 929-936, doi: 10.1056/NEJMoa2001191, indexed in Pubmed: 32004427.

10. Sikorska K. Coronavirus Disease 2019 as a challenge for maritime medicine. Int Marit Health. 2020; 71(1): 4, doi: 10.5603/ IMH.2020.0002, indexed in Pubmed: 32212138.

11. https://www.cdc.gov/quarantine/maritime/recommendations-for-ships.html (June 28, 2020). 